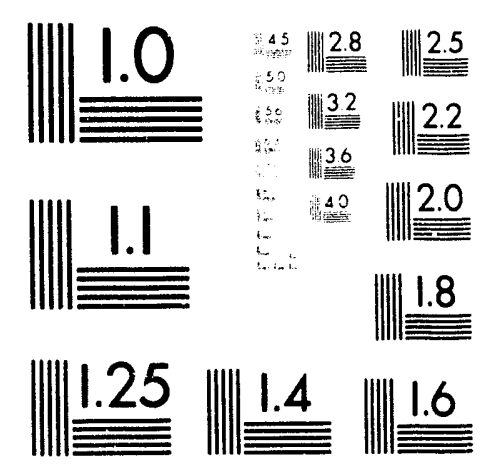



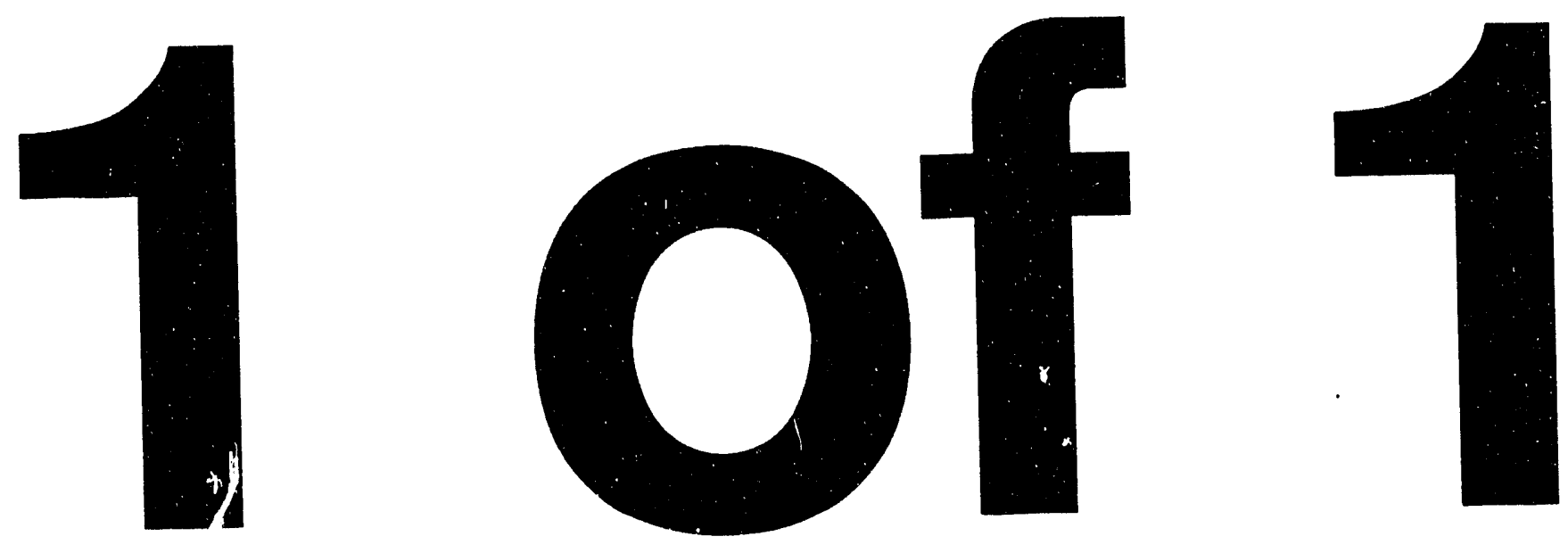


\title{
An Ion-Source Model for First-Order Beam Dynamic Codes
}

\author{
C. L. Fink, and B. P. Curry \\ Argonne National Laboratory \\ 9700 S. Cass Avenue \\ Argonne, II 60439
}

\section{Abstract}

A model of a plasma ion source has been developed that approximates the system of Poisson and Boltzman-Vlasov equations normally used to describe ion sources by an extemal electric field, a collective electric field due to the charge column, and the starting boundary conditions. The equations of this model can be used directly in the Lorentz force equation to calculate trajectories without iteration.

\section{INTRODUCTION}

The physics of a plasma ion-source is usually described in terms of a system of Poisson and Boltzman-Vlasov equations for each of the ion species involved ${ }^{l}$. This system is usually solved numerically after various levels of approximation are made to make the problem tractable. In general, this procedure gives reasonable agreement with experiment, especially for positive ion codes. The problem, however, is that the effects of various approximations and/or source components on source performance are difficult to separate and thus optimization of source performance is difficult.

In this paper, we develop a model of an ion source that consists of an external electric field due primarily to the source geometry, a collective electric field due to the charge column, and a set of initial boundary conditions on the ions as they are injected into the external and collective fields. Since the charge column is not infinitely long, there will be axial component to the collective electric field 2 . Because this model separates out the external and collective fields, it should be relatively easy to trace particle trajectories or incorporate the model into codes like TRACE2D ${ }^{3}$. In this paper, we will discuss the key features and assumptions of the model. We will provide a more detailed comparison of the model predictions with those of standard source codes such as $\mathrm{SNOW}^{4}$ in a later paper.

\section{OVERVIEW OF A TYPICAL POSITIVE-ION SOURCE MODEL}

Figure 1 shows the simple positive-ion source geometry to be modeled. The plasma to extractor electrode distance $D$ is $8 \mathrm{~mm}$, the aperture radius is $2.5 \mathrm{~mm}$, and the sheath to plasma electrode distance $\mathrm{L}$ varies between 1 and $4 \mathrm{~mm}$. Note that the usual hole in the extractor electrode has been eliminated for simplicity.

In source codes like SNOW, the initial ion beam is injected into the plasma with an energy of 5 to $10 \mathrm{eV}$ in a direction parallel to the axis with a current density Jo. These positive ions are neutralized within the plasma by a Boltzman distribution of electrons with an electron temperature typically of 2 to $10 \mathrm{eV}$. As the ions approach the plasma electrode a sheath forms at the edge of the plasma, which has the effect of reflecting the electrons and accelerating the positive ions out of the plasma. Usually, the width of the sheath is approximately 0.1 to $0.001 \mathrm{~mm}$ with an electric field of a few $\mathrm{MV} / \mathrm{m}$. After being extracted from the sheath the ions are further accelerated by the electric fivid from the extractor electrode.

\section{SOURCE GEOMETRY}

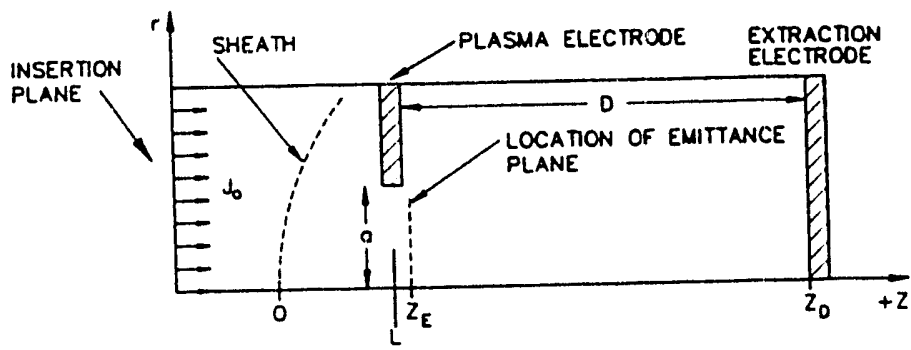

Figure 1. Definition of Source Geometry

The Poisson and Boltzman-Vlasov equations are solved iteratively by calculating an initial potential distribution, raytracing the ion trajectories to obtain a new charge density, and then solving the Poisson equation until consistency is obtained. This final potential is then used to ray-trace the ion to obtain current densities and two-dimensional phase distribution at the location $\mathrm{Z}_{\mathrm{E}}$.

\section{CALCULATION OF EXTERNAL FIELD}

The external field is determined by solving Laplace's equation for the source geometry using a code such as SIMION or by using the technique of aperture superposition 5 . The problem in determining the external fields arises from the fact that the this field depends on the unknown position and shape of the sheath formed in extracting the plasma.

In our model we approach this problem by assuming that the position of the sheath has the dominant effect on determining the external fields and that the shape of the sheath is less important. The top half of Fig. 2 shurws calculations ${ }^{6}$ of the phase-stace distribution from SNOW calculations for a range of current densities using the geometry of Fig. 1. The bottom half shows phase-space

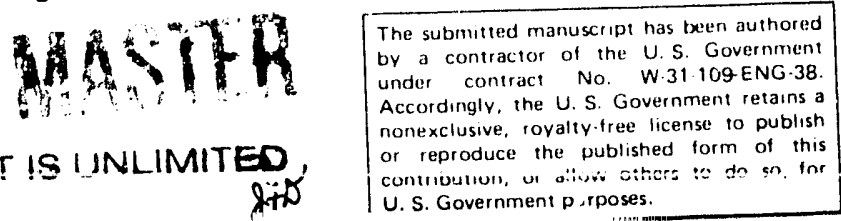


distributions calculated using external fields for a planar plate located at various values of $L$ relative to the plasma electrode. The key observation from these calculations is that the shape of the phase-space distributions does not appear to be strongly dependent on the space-charge forces, and that the current density Jo is closely related to the sheath position.
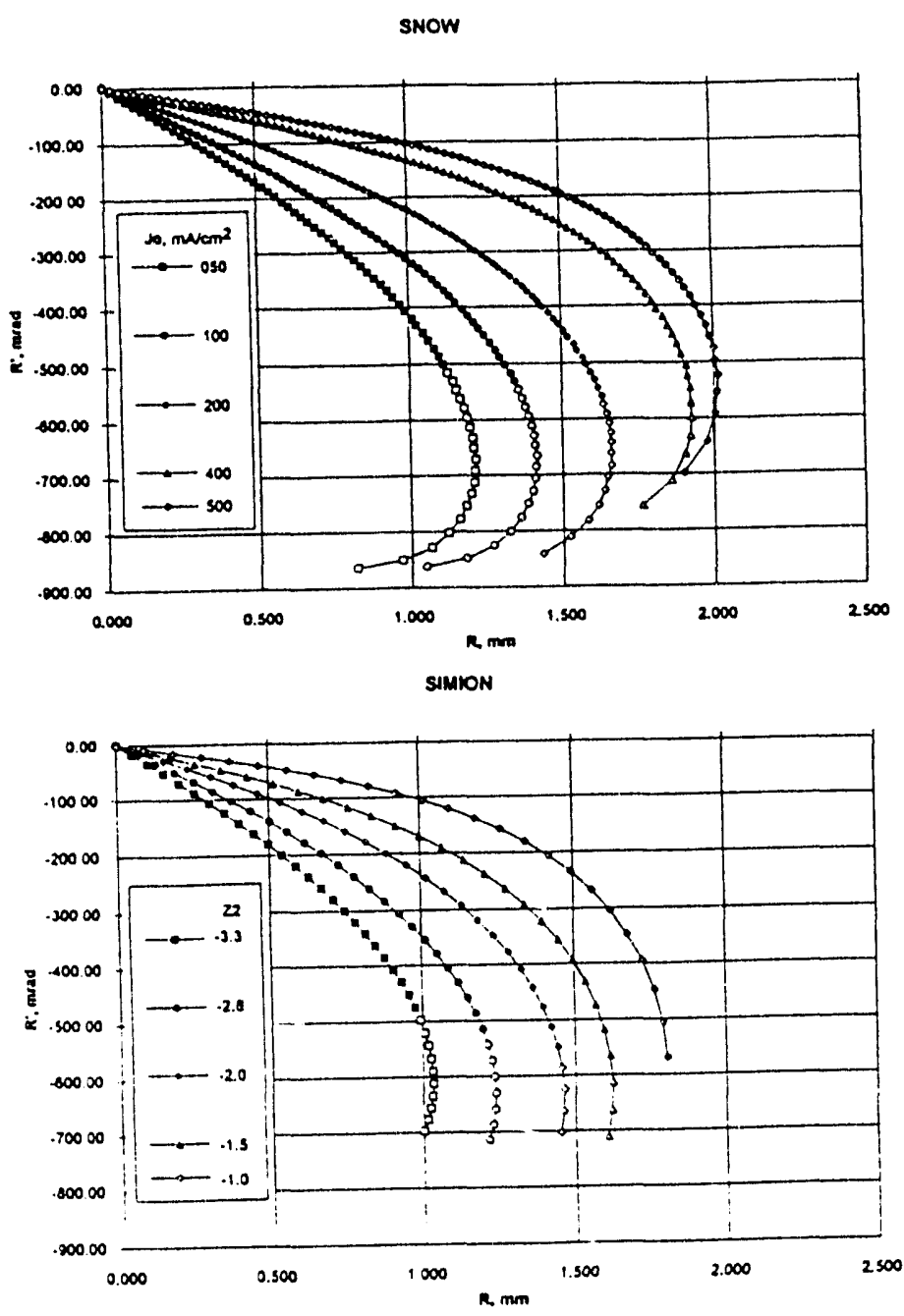

Figure 2. Phase-Space Distributions

The preceding observations suggest that a good first approximation in calculating the external fields is to simply replace the sheath by a planar plate and to choose the position of the plate to provide the desired current density. Using a sheath shape that is not planar does not complicate the analytical determination of the external fields. The complication is in devising a method to determine the sheath shape.

The key is to determine the functional relationship between Jo and the plate position without solving the Poisson-Boltzman equations. We propose that this relationship can be determined by requiring that the axial ciectric field die to the charge column be equal to the
Laplacian electric field when the sheath is replaced by a conducting surface. Figure 3 shows a plot of the curreat density versus sheath-to-plasma eloctrode distance L. The solid line shows the sheath position (5-eV equipotential) from the SNOW calculation. The open symbols show the current densities required to match the Laplacian and column eloctric fields at a particular $L$ using a planar emission eloctrode The column electric fields viere calculated using the fonnulas for a column of charge of constant radius $a$ as described in the following section. The data suggest that the exact current density for a particular $L$ is not a sensitive function of $a$. The use of the experimental aperture size of $2.5 \mathrm{~mm}$ appears to be a good initial choice to reproduce the SNOW data. The solid symbols correspond to a concave emission electrode and appear to give a better fit to the data over the range of $L$. The problem in concave calculations is in determining the radius of curvature of the emission electrode.

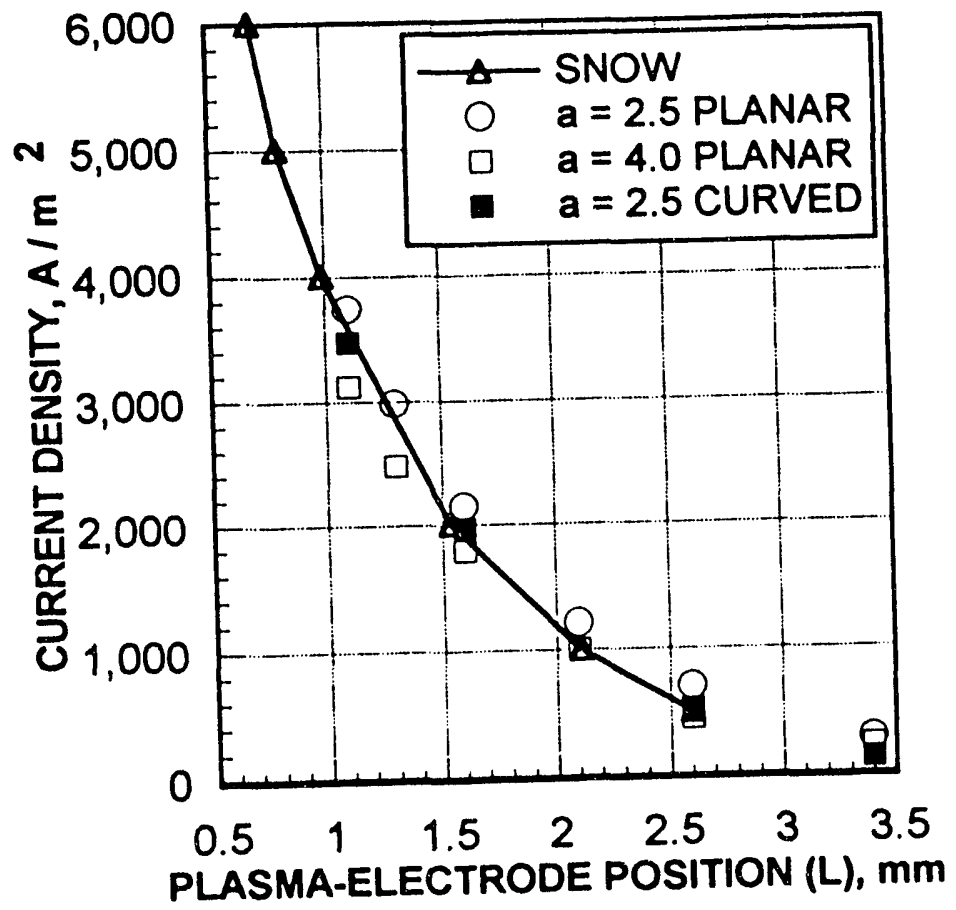

Figure 3. Current Density Versus Sheath Position

\section{BOUNDARY CONDITIONS}

One of the most uncertain features of this model is the boundary conditions to use at the emission electrode. Since the sheath region is relatively thin and since the sheath shape is approximately planar, it would not be unreasonable to expect that the ions would not be significantly bent in the sheath region and that the amount of energy gain would be limited. Thus a first approximation may be simply to assume that the ions have the same direction and energy at the sheath as at the insertion plane. Note, however, that if the ions are not emerging perpendicular to the emission surface, then the energy of the emitted ions can have a significant effect on the resulting beam optics. 


\section{ELECTRIC FIELD FROM A COLUMN OF CHARGE}

The potential due to a distribution of space charge is given by Kirstein et. $\mathrm{al}^{7}$. This potential vanishes at $\mathrm{z}=0$, but not at $z_{D}$. If we add a potential that is a linear function of $z$, and that varies from 0 to the negative of Kirstein's potential at $z_{D}$, then we will also have zero potential at the end of the column. Differentiating the modified potential gives the space charge field.

$E z(0, z)=\frac{1}{2 \varepsilon_{0}}\left\{\int_{0}^{z} \rho\left(z^{\prime}\right)\left[\frac{z+z^{\prime}}{\sqrt{a^{2}+\left(z+z^{\prime}\right)^{2}}}-\frac{z-z^{\prime}}{\sqrt{a^{2}+\left(z-z^{\prime}\right)^{2}}}\right] d z^{\prime}\right.$

$+\int_{z}^{z} \rho\left(z^{\prime}\right)\left[\frac{z+z^{\prime}}{\sqrt{a^{2}+\left(z+z^{\prime}\right)^{2}}}-\frac{z-z^{\prime}}{\sqrt{a^{2}+\left(z-z^{\prime}\right)^{2}}}-2\right] d z^{\prime}$

$\left.+\frac{1}{z_{D}} \int_{0}^{z_{p}} \rho\left(z^{\prime}\right)\left[\sqrt{a^{2}+\left(z_{D}-z^{\prime}\right)^{2}}-\sqrt{a^{2}+\left(z_{D}+z^{\prime}\right)^{2}}+2 z^{\prime}\right] d z^{\prime}\right\}$

where $a$ is the column radius and $z_{D}$ is its length. In this equation we have assumed the charge density is uniform in the radial direction and $a$ is constant.

We can use the above expression to obtain the radial and axial field components off the axis, using the paraxial approximation:

$$
\begin{aligned}
& E_{r}(r, z)=\frac{r}{2}\left[\frac{\rho(z)}{\varepsilon_{0}}-\frac{\partial}{\partial z} E_{z}(0, z)\right] \\
& E_{z}(r, z)=E_{z}(0, z)-\frac{r^{2}}{2}\left[\frac{\partial^{2}}{\partial^{2}}\left(E_{z}(0, z)\right)-\frac{1}{\varepsilon_{0}} \frac{\partial}{\partial z}(\phi(z))\right]
\end{aligned}
$$

Inserting the axial space charge field (eq. (1)) into eqs (2) and (3) yields the entire space charge field in the paraxial approximation., provided the charge density is known.

The charge density is related to the ion beam current density by

$\rho\left(z^{\prime}\right)=\frac{4}{9} \frac{J_{0}}{\chi} \frac{\varepsilon_{0}}{\sqrt{T_{0}+\varphi\left(z^{\prime}\right)}}$

where $T_{0}$ is the ions' initial kinetic energy, $\chi=(+/ 9) \varepsilon_{0} \sqrt{2 e / m_{\text {ion }}}, \mathrm{J}_{0}$ is the team current density, and $\phi$ is the total potential (solution of Poisson's equation) on axis. We approximate this potential with the space charge limited planar diode potential given by

$$
\lambda^{\prime}\left(z^{\prime}\right)=\left(\frac{J_{0}}{x}\right)^{2 / 3} z^{-4 / 3}
$$

Now we can relate the sheath position to the beam current density. Since the sheath is an emitting surface, the total field (applied plus space charge) must vanish at $z=0$. The space charge field is obtained from eq. (1).

$$
\begin{aligned}
& E z(0,0)=\frac{1}{\varepsilon_{0}} \int_{0}^{z_{D}} \rho\left(z^{\prime}\right)\left\{\frac{z^{\prime}}{z_{D}}-1+\frac{z^{\prime}}{\sqrt{a^{2}+z^{\prime 2}}}\right. \\
& \left.-\frac{1}{2 z_{D}}\left(\sqrt{a^{2}+\left(z_{D}+z^{\prime}\right)^{2}}-\sqrt{a^{2}+\left(z_{D}-z^{\prime}\right)^{2}}\right)\right\} d z^{\prime}
\end{aligned}
$$

Equating this field to the negative of the field resulting from Laplace's equation, using eqs. (4) and (5), gives the beam current density as a function of the applied voltages, the sheath location, and the extraction geometry.

\section{DISCUSSION}

Using the assumptions and formulas presented in this paper, it is relatively easy to calculate ion trajectories from an ion source without having to solve iteratively a complicated system of Poisson and Boltzman-Vlasov equations. In addition the physic issues of the problem are more readily apparent. We are currently in the process of developing a code using the model described in this paper to calculate phase-space distributions for comparison with SNOW calculations. It is expected that by varying the various parameters and assumptions in our model to obtain agreement with the SNOW data, we will obtain additional information on which source parameters are limiting source performance.

\section{REFERENCES}

[1] J. H. Whealton, et al., "Computer Modeling of Negative Ion beam Formation," J. Appl. Phys. 64, 6210 (1988)

[2] D. A. De Wolf, "Basics of Electron Optics," John Wiley\&Sons, Inc. 1990.

[3] K. R. Crandall and D. P. Rusthoi, "Documentation for TRACE: An Interactive Beam-Transport Code," Los Alamos keport LA10235-MS, (1985)

[4] J. E. Boers, "SNOW", Thunderbird Simulations, 626 Bradsfield Drive, Garland Texas, 75042-6005

[5] P. W. Hawkes, E. Kasper, "Principles of Electron Optics," Academic Press, Volume 1, 143 (1989)

[6] C. L. Fink, and B. P. Curry, "Simple Optics Description of The Plasma Sheath and Plasma Electrode Region," Sixth Intern. Symp. on Production and Neutralization of Negative lons and Beams, Brookhaven, Nov. 1992

[7] P. T. Kirstein, et al., "Space-Che rge Flow," McGraw-Hill, New York 1967 


\section{DISCLAIMER}

This report was prepared as an account of work sponsored by an agency of the This reportes Government. Neither the United States Goverameret nor any agency United sor any of their employees, mikes any waranty, eppress or implied, or thereof. dor any of their employecs, mility for the accoracy, compieteness. or use-

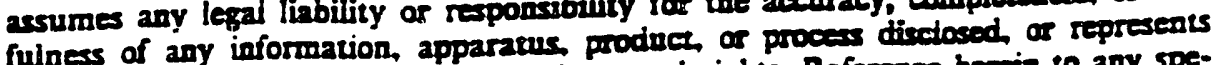

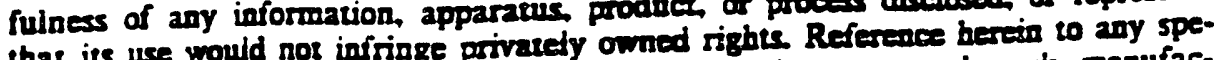
thas its use would not infringe privately owned rights Refercece hereso to any speciric commercial produch process, or semvice by trade andy its endorsement recom.

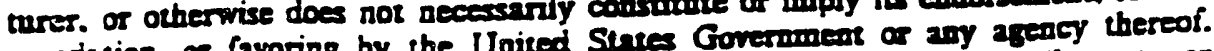
mendation. or favoring by the United States Goverim do not necescarily state or

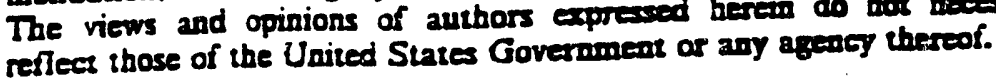



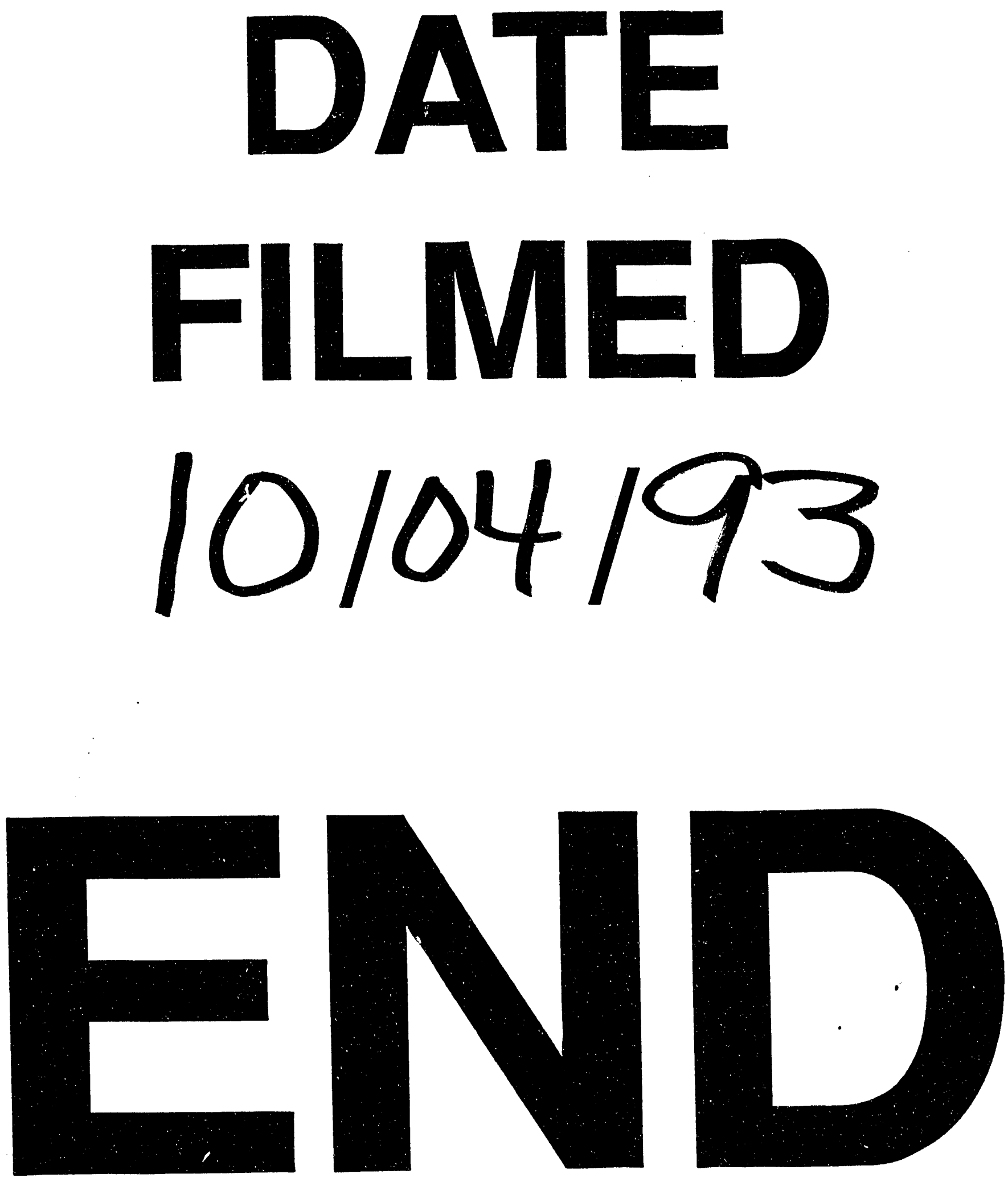
